\title{
A variational inequality arising from optimal surrender of variable annuity with lookback benefit
}

\author{
Junkee Jeon ${ }^{1}$ and Minsuk Kwak ${ }^{2 *}$ (i)
}

\section{"Correspondence:}

mkwak@hufs.ac.kr

${ }^{2}$ Department of Mathematics,

Hankuk University of Foreign

Studies, Yongin, Korea

Full list of author information is

available at the end of the article

\section{Springer}

\begin{abstract}
We introduce a variable annuity (VA) contract with a surrender option and lookback benefit, that is, the benefit of the VA contract is linked to the maximum process of the policyholder's account value. In contrast to the constant guarantee model provided in Bernard et al. (Insur. Math. Econ. 55:116-128, 2014), it is optimal for the policyholder of the VA contract with lookback benefit to surrender the VA contract when the policyholder's account value is below or equal to the optimal surrender boundary. Thus, from the perspective of the insurer to construct a portfolio of VA contracts, utilizing the VA contracts with lookback benefit along with VA contracts with constant guarantee provides the diversification of early surrenders. The valuation of this contract can be described as a two-dimensional parabolic variational inequality. By converting this into the one-dimensional problem, we obtain the integral equations for the value function and the free boundary. The recursive integration method is applied to obtain the numerical solutions. We also provide comparative statics of the optimal surrender boundaries with respect to various parameters.
\end{abstract}

Keywords: Variable annuities; Optimal surrender; Lookback benefit; Free boundary problem; Integral equation

\section{Introduction}

Traditional annuity contracts offer a fixed amount of post-retirement income. In contrast, by investing the insurance premium in the equity market, variable annuity (VA) contracts provide random payoff, which depends on the financial market environment. At the same time, VA contracts offer various types of minimum guarantees for the case of poor performance of the equity market. Thus, a policyholder of the VA contract may enjoy the upside opportunity in the equity market while having certain protection against the downside risk.

The protection against the downside risk in a VA contract is not given for free. A stream of the fee is collected periodically from the policyholder's account, and these fees are utilized by the insurer to construct a hedge portfolio for the guaranteed payoff of the contract. A VA contract usually incorporates a surrender option, which allows the policyholder to terminate the VA contract before maturity, receiving a pre-specified surrender benefit. In-

(c) The Author(s) 2022. This article is licensed under a Creative Commons Attribution 4.0 International License, which permits use sharing, adaptation, distribution and reproduction in any medium or format, as long as you give appropriate credit to the original author(s) and the source, provide a link to the Creative Commons licence, and indicate if changes were made. The images or other third party material in this article are included in the article's Creative Commons licence, unless indicated otherwise in a credit line to the material. If material is not included in the article's Creative Commons licence and your intended use is not permitted by statutory regulation or exceeds the permitted use, you will need to obtain permission directly from the copyright holder. To view a copy of this licence, visit http://creativecommons.org/licenses/by/4.0/. 
deed, the optimal surrender strategy should be carefully considered to calculate the fair fee rate correctly. In line with this, we investigate the optimal surrender strategy and the value of a VA contract, in which the payoff of the VA contract is associated with the maximum value process of the policyholder's account value.

There have been several studies on the optimal surrender strategies for VA contracts recently, such as Bernard et al. [1], Shen et al. [2], Kang and Ziveyi [3], and Jeon and Kwak $[4,5]$. In the baseline model of Bernard et al. [1], the payoff at the maturity is the maximum of the policyholder's accumulated account value and a constant minimum guarantee. If the policyholder surrenders the contract before maturity, the surrender benefit is the accumulated account value with some penalty proportional to the account value. In Shen et al. [2], the modeling of the surrender benefit is different from Bernard et al. [1]. When the policyholder surrenders the VA contract before the maturity in Shen et al. [2], the policyholder receives the underlying account value at the maturity as well as the embedded option payoff at the surrender time with a penalty in the payoff of the embedded option. Kang and Ziveyi [3] extend the baseline model of Bernard et al. [1] to the model with stochastic volatility and stochastic interest rate. They derive a 4-dimensional freeboundary partial differential equation (PDE) and solve it using the method of lines (MOL) approach. As an extension of Shen et al. [2], Jeon and Kwak [4] study the optimal surrender strategies and valuations of variable annuities in which the embedded guarantees can be path-dependent.

Recently, Jeon and Kwak [5] investigated the VA contract with surrender guarantee. In contrast to Bernard et al. [1], the surrender benefit in their study is linked to the maximum value between the policyholder's account value and the guaranteed minimum accumulated benefit. Moreover, they show that the results in Jeon and Kwak [5] can be reduced to those in Bernard et al. [1] under certain parameter conditions. The model in this paper can be considered as an extension of Jeon and Kwak [5] because the accumulated account value in the surrender benefit is replaced by a constant multiple of the maximum process of the policyholder's account value.

Our model can be defined as an optimal stopping problem or a free boundary problem that takes into account the maximum process of the policyholder's account process in the payoff function. The solution of this optimal stopping problem satisfies 2-dimensional parabolic variational inequality. ${ }^{1}$ In the option pricing theory, there are many free boundary problems considering the maximum process of the underlying asset, such as the Russian option in Peskir [8] or the American floating strike lookback option in Dai and Kwok [9]. These problems can be reduced to a 1-dimensional problem through proper spatial transforms. However, our model is challenging to solve because we cannot apply usual transforms in Peskir [8] and Dai and Kwok [9] to our problem directly due to the constant minimum guarantee.

Our contribution is to suggest an idea to convert our problem into a 1-dimensional problem. Specifically, we transform our problem in two steps. First, we change our original 2dimensional problem (Variational Inequality 1 ) to the other 2-dimensional problem (Variational Inequality 2) by introducing a new state variable that incorporates the maximum process of the underlying asset and the constant minimum guarantee simultaneously. Second, we can reduce the transformed 2-dimensional problem (Variational Inequality 2) into

${ }^{1}$ We refer to Jolaoso et al. [6] and Liu et al. [7] as recent studies on variational inequalities. 
the 1-dimensional problem (Variational Inequality 3). Moreover, on the basis of the Mellin transform techniques, we derive the integral equations for the value function and the free boundary of the reduced 1-dimensional problem. To the best of our knowledge, no research of the two-dimensional variational inequality we are dealing has been carried out.

The 1-dimensional variational inequality we obtained as a result of two-step transformation is associated with an optimal stopping problem that is equivalent to an early exercise of some type of American option. ${ }^{2}$ Thus, we can apply well-developed techniques to our optimal stopping problem. In particular, we apply the integral representation of an American option value (see Carr et al. [11], Detemple [12], Kim [13]) and derive the value of the VA contract in analytic form. To obtain a specific solution, we apply the recursive integration method proposed by Huang et al. [14], solving the integral equation numerically. Jeon and Kwak $[4,5]$ show the efficiency and accuracy of RIM by comparing benchmark values.

The remainder of the paper is organized as follows. We introduce the model in Sect. 2; the method and solution are provided in Sect. 3. In Sect. 4, we present the properties of the optimal surrender strategy with numerical examples. Section 5 is the concluding remark of the paper.

\section{Model formulation}

$V A$ contract We assume that the policyholder pays a single premium $F_{0}$ to initiate the contract at time 0 . Then the policyholder's premium is invested in a fund or an index $S_{t}$ that satisfies the following stochastic differential equation (SDE) under the risk-neutral measure $\mathbb{Q}$ :

$$
d S_{t}=r S_{t} d t+\sigma S_{t} d W_{t}
$$

where $r>0$ and $\sigma>0$ are the constant risk-free interest rate and the constant volatility of $S_{t}$, respectively. Also, $W_{t}$ is a standard Brownian motion on filtered probability space $(\Omega, \mathbb{F}, \mathbb{Q})$, where $\mathbb{F}=\left(\mathcal{F}_{t}\right)_{t \geq 0}$ is the natural filtration generated by $\left(W_{t}\right)_{t \geq 0}$.

The policyholder has to continuously pay a certain amount of insurance fee at the cost of the insurance company managing the underlying account. We assume that the insurance company withdraws a percentage $c$ from the policyholder's account. Then, at any time $t$, we have the following relationship between the policyholder's account $F_{t}$ and underlying fund $S_{t}$

$$
\frac{F_{t}}{F_{0}}=e^{-c t} \frac{S_{t}}{S_{0}}
$$

and thus the dynamics of $F_{t}$ is given by

$$
d F_{t}=(r-c) F_{t} d t+\sigma F_{t} d W_{t}
$$

under $\mathbb{Q}$ measure.

${ }^{2}$ The valuation of a defined benefit retirement pension plan that allows early retirement considered in Bian et al. [10] is also an example that falls into this category. 


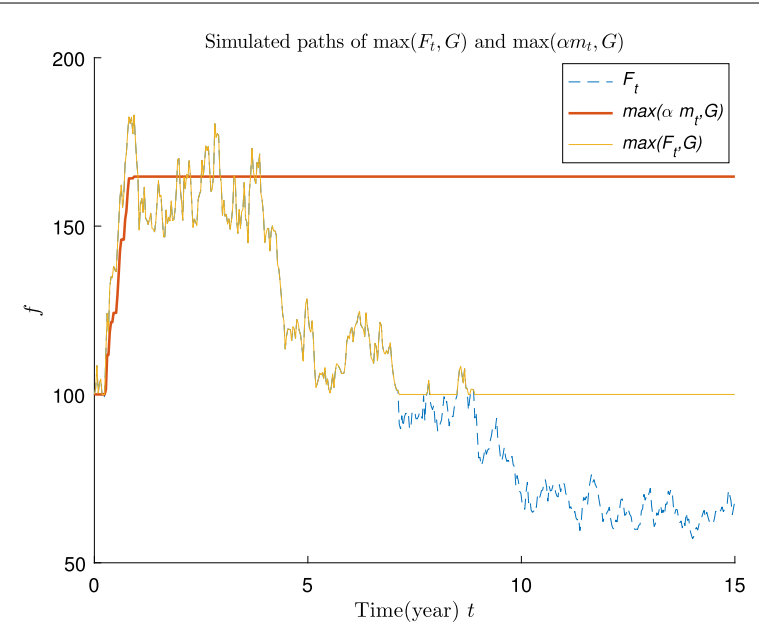

Figure 1 Simulated path of $m_{t}$ and $F_{t}$. Parameter values are given by $r=0.05, c=0.03, \sigma=0.2, F_{0}=100$, $G=100, \alpha=0.9$ and $T=15$

For the policyholder's account value $F_{t}$, we define the maximum process $m_{t}$ of $F_{t}$ as

$$
m_{t}=\max _{0 \leq \gamma \leq t} F_{\gamma}
$$

Now, we consider a VA contract with lookback benefit. More precisely, at the maturity of contract $T$, the policyholder will receive $\max \left(\alpha m_{T}, G\right)$, where $m_{T}=\max _{0 \leq \gamma \leq T} F_{\gamma}$ is the maximum of the account value during $[0, T]$, and $G$ is a constant guarantee at time $T$. Here, $\alpha$ is a positive constant with $0<\alpha \leq 1$. For $\alpha$ near 1 , this type of VA contract may be attractive to policyholders who want more benefit, as shown in Fig. 1, although it may be more expensive.

Surrender option As in the previous studies on the optimal surrender of variable annuities (e.g., Bernard et al. [1], Shen et al. [2], Kang and Ziveyi [3], Jeon and Kwak [4, 5]), the policyholder is able to surrender the VA contract at any time $t$ before the maturity $T$. Then, the surrender benefit of the VA contract is equal to

$$
\left(1-p_{t}\right) \max \left(\alpha m_{t}, G\right)
$$

where $p_{t}$ is the penalty percentage charged when surrendering the contract at time $t$. As in Bernard et al. [1], we assume that

$$
p_{t}=1-e^{-\kappa(T-t)}
$$

for $0 \leq \kappa<r$ so that the surrender benefit is given by

$$
e^{-\kappa(T-t)} \max \left(\alpha m_{t}, G\right)
$$

Remark 2.1 If $\kappa \geq r$, the early surrender penalty is too severe, so that early surrender is always sub-optimal, and it is optimal to wait until maturity. Thus, we assume that $0 \leq \kappa<r$ throughout the paper. 
Table 1 Comparison of VA benefits between three models

\begin{tabular}{lll}
\hline Model & Surrender at time $t$ & At maturity $T$ \\
\hline Bernard et al. [1] & $e^{-\kappa(T-t)} F_{t}$ & $\max \left(F_{T}, G\right)$ \\
Jeon and Kwak [5] & $e^{-\kappa(T-t)} \max \left(F_{t}, G\right)$ & $\max \left(F_{T}, G\right)$ \\
Our model & $e^{-\kappa(T-t)} \max \left(\alpha M_{t}, G\right)$ & $\max \left(\alpha M_{T}, G\right)$ \\
\hline
\end{tabular}

As mentioned in the introduction, the VA benefit of our model is different from that of Bernard et al. [1] or Jeon and Kwak [5]. We summarize this difference in Table 1.

Then, the concern is finding the optimal time for the policyholder to surrender the VA contract, and it is an optimal stopping problem.

Let $V(t, f, m)$ be the value of our VA contract at time $t$ before surrender with $F_{t}=f$ and $m_{t}=m$. Then, in the absence of arbitrage opportunities, the value function $V(t, f, m)$ is a solution of the following optimal stopping problem

$$
V(t, f, m)=\sup _{t \leq \theta \leq T} e^{-r(\theta-t)} \mathbb{E}\left[e^{-\kappa(T-\theta)} \max \left(\alpha m_{\theta}, G\right) \mid F_{t}=f, m_{t}=m\right]
$$

where $\theta$ is the stopping time of the filtration $\mathbb{F}$, and the conditional expectation $\mathbb{E}\left[\cdot \mid \mathcal{F}_{t}\right]$ is calculated under the risk-neutral measure $\mathbb{Q}$.

\section{Solution of free boundary problems}

By relying on a standard approach of optimal stopping problem (see Peskir [8]) in (1), we derive the following Variational Inequality 1 (VI 1):

\section{Variational Inequality 1}

$$
\left\{\begin{array}{l}
\mathcal{L} V(t, f, m) \leq 0, \quad \text { if } V(t, f, m)=e^{-\kappa(T-t)} \max (\alpha m, G), \\
\mathcal{L} V(t, f, m)=0, \quad \text { if } V(t, f, m)>e^{-\kappa(T-t)} \max (\alpha m, G), \\
V(T, f, m)=\max (\alpha m, G) \quad \text { and } \quad \frac{\partial V}{\partial m}(t, m, m)=0,
\end{array}\right.
$$

on region $\mathcal{R} \equiv\{(t, f, m) \mid 0 \leq t \leq T, 0<f \leq m, 0<m<\infty\}$ and the operator $\mathcal{L}$ is

$$
\mathcal{L} \equiv \frac{\partial}{\partial t}+\frac{\theta^{2}}{2} f^{2} \frac{\partial^{2}}{\partial f^{2}}+(r-c) f \frac{\partial}{\partial f}-r .
$$

Let $\mathbf{C}$ and $\mathbf{S}$ denote the continuation region and the surrender region of VI $\mathbf{1}$, respectively. In terms of value function $V(t, f, m), \mathbf{C}$ and $\mathbf{S}$ are defined by

$$
\begin{aligned}
& \mathbf{C}=\left\{(t, f, m) \in \mathcal{R} \mid V(t, f, m)>e^{-\kappa(T-t)} \max (\alpha m, G)\right\}, \\
& \mathbf{S}=\left\{(t, f, m) \in \mathcal{R} \mid V(t, f, m)=e^{-\kappa(T-t)} \max (\alpha m, G)\right\} .
\end{aligned}
$$

The boundary that separates $\mathbf{C}$ from $\mathbf{S}$ is referred to as the optimal surrender boundary or free boundary and is given by

$$
B(t, m)=\sup \left\{f \in \mathbb{R}_{+} \mid(t, f, m) \in \mathbf{S}\right\} .
$$

To solve VI 1, we consider the following Variational Inequality 2 (VI 2): 


\section{Variational Inequality 2}

$$
\left\{\begin{array}{l}
\mathcal{L} V^{\star}(t, f, M) \leq 0, \quad \text { if } V^{\star}(t, f, M)=e^{-\kappa(T-t)} \alpha M \\
\mathcal{L} V^{\star}(t, f, M)=0, \quad \text { if } V^{\star}(t, f, M)>e^{-\kappa(T-t)} \alpha M \\
V^{\star}(T, f, M)=\alpha M \quad \text { and } \quad \frac{\partial V^{\star}}{\partial M}(t, M, M)=0
\end{array}\right.
$$

on region $\mathcal{R}^{\star} \equiv\{(t, f, M) \mid 0 \leq t \leq T, 0<f \leq M, 0<M<\infty\}$.

Proposition 3.1 On region $\mathcal{R}=\{(t, f, m) \mid 0 \leq t \leq T, 0<f \leq m, 0<m<\infty\}, V(t, f, m)=$ $V^{\star}(t, f, M)$ with $M=\max \left(m, \frac{G}{\alpha}\right)$ is a unique solution of VI 1.

Proof We will use the idea similar to Theorem 4.1 in Buchen and Konstandatos [15]. Note that

$$
\max (\alpha m, G)=\max \left(\alpha \max \left(m, \frac{G}{\alpha}\right), G\right) .
$$

Then, since $M$ is independent of $t, f$, and $V^{\star}(t, f, M)$ is the solution of VI 2 , we can easily obtain that

$$
\left\{\begin{array}{l}
\mathcal{L} V^{\star}\left(t, f, \max \left(m, \frac{G}{\alpha}\right)\right) \leq 0, \quad \text { if } V^{\star}\left(t, f, \max \left(m, \frac{G}{\alpha}\right)\right)=e^{-\kappa(T-t)} \max \left(\alpha \max \left(m, \frac{G}{\alpha}\right), G\right), \\
\mathcal{L} V^{\star}\left(t, f, \max \left(m, \frac{G}{\alpha}\right)\right)=0, \quad \text { if } V^{\star}\left(t, f, \max \left(m, \frac{G}{\alpha}\right)\right)>e^{-\kappa(T-t)} \max \left(\alpha \max \left(m, \frac{G}{\alpha}\right), G\right), \\
V^{\star}\left(T, f, \max \left(m, \frac{G}{\alpha}\right)\right)=\max \left(\alpha \max \left(m, \frac{G}{\alpha}\right), G\right)
\end{array}\right.
$$

on region $\left\{(t, f, m) \mid 0 \leq t \leq T, 0<f \leq \max \left(m, \frac{G}{\alpha}\right), 0<m<\infty\right\}$, which includes the region $\mathcal{R}$.

If $m \leq \frac{G}{\alpha}$, then $M=\frac{G}{\alpha}$. Thus, $\frac{\partial V^{\star}}{\partial m}(t, m, M)=0$ for this case. Otherwise, if $m>\frac{G}{\alpha}$, then $M=m$. Thus, we have

$$
\frac{\partial V^{\star}}{\partial m}(t, m, M)=\left.\frac{\partial V^{\star}}{\partial m}(t, f, M)\right|_{f=m}=\left.\frac{\partial V^{\star}}{\partial M}(t, f, M)\right|_{f=M}=0 .
$$

Thus, $V^{\star}\left(t, f, \max \left(m, \frac{G}{\alpha}\right)\right)$ satisfies VI 1.

According to the partial differential equation (PDE) theory of variational inequality, the (strong) solutions of the VI 1 and VI 2 uniquely exist (see Friedman [16, 17]). So, we can conclude that $V(t, f, m)=V^{*}\left(t, f, \max \left(m, \frac{G}{\alpha}\right)\right)$ is a unique solution of VI 1 on region $\mathcal{R}$.

The region $\mathcal{R}$ can be divided into continuation region $\mathbf{C}^{\star}$ and surrender region $\mathbf{S}^{\star}$ defined as

$$
\begin{aligned}
& \mathbf{C}^{\star}=\left\{(t, f, M) \in \mathcal{R}^{\star} \mid V^{\star}(t, f, M)>e^{-\kappa(T-t)} \alpha M\right\}, \\
& \mathbf{S}^{\star}=\left\{(t, f, M) \in \mathcal{R}^{\star} \mid V^{\star}(t, f, M)=e^{-\kappa(T-t)} \alpha M\right\},
\end{aligned}
$$

respectively.

Here, $B^{\star}(t, M)$ is the optimal surrender boundary of VI 2 given by

$$
B^{\star}(t, M)=\sup \left\{f \in \mathbb{R}_{+} \mid(t, f, M) \in \mathbf{S}^{\star}\right\} .
$$


Proposition 3.2 The optimal surrender boundaries $B(t, m)$ and $B^{\star}(t, M)$ satisfy the following relationship:

$$
B(t, m)=B^{\star}\left(t, \max \left(m, \frac{G}{\alpha}\right)\right) .
$$

Proof If $(t, f, m) \in \mathbf{S}$, then

$$
V(t, f, m)=e^{-\kappa(T-t)} \max (\alpha m, G)=e^{-\kappa(T-t)} \alpha \max \left(m, \frac{G}{\alpha}\right),
$$

and $V(t, f, m)=V^{\star}\left(t, f, \max \left(m, \frac{G}{\alpha}\right)\right)$ in $\mathbf{S}$ by Proposition 3.1. Thus, $V^{\star}\left(t, f, \max \left(m, \frac{G}{\alpha}\right)\right)=$ $e^{-\kappa(T-t)} \alpha \max \left(m, \frac{G}{\alpha}\right)$ in $\mathbf{S}$, and this implies that $\left(t, f, \max \left(m, \frac{G}{\alpha}\right)\right) \in \mathbf{S}^{\star}$ when $(t, f, m) \in \mathbf{S}$.

Similarly, if $\left(t, f, \max \left(m, \frac{G}{\alpha}\right)\right) \in \mathbf{S}^{\star}$, we can obtain $(t, f, m) \in \mathbf{S}$. Hence,

$$
(t, f, m) \in \mathbf{S} \Longleftrightarrow\left(t, f, \max \left(m, \frac{G}{\alpha}\right)\right) \in \mathbf{S}^{\star} .
$$

By the definition of optimal surrender boundaries,

$$
B(t, m)=B^{\star}\left(t, \max \left(m, \frac{G}{\alpha}\right)\right) .
$$

Remark 3.1 From VI 1 and VI 2, we have the following smooth-pasting conditions.

$$
\begin{aligned}
& V(t, B(t, m), m)=e^{-\kappa(T-t)} B(t, m), \\
& \frac{\partial V}{\partial m}(t, B(t, m), m)=0 .
\end{aligned}
$$

and

$$
\begin{aligned}
& V^{\star}\left(t, B^{\star}(t, M), M\right)=e^{-\kappa(T-t)} B^{\star}(t, M), \\
& \frac{\partial V^{\star}}{\partial M}\left(t, B^{\star}(t, M), M\right)=0 .
\end{aligned}
$$

We can easily confirm that Proposition 3.2 does not violate the above smooth-pasting conditions.

Let us consider the following substitution:

$$
z=\frac{f}{M}, \quad Q(t, z)=\frac{V^{\star}(t, f, M)}{M} .
$$

Then, VI 2 can be converted to the following Variational Inequality 3 (VI 3):

\section{Variational Inequality 3}

$$
\left\{\begin{array}{l}
\mathcal{L} Q(t, z) \leq 0, \quad \text { if } Q(t, z)=e^{-\kappa(T-t)} \alpha, \\
\mathcal{L} Q(t, z)=0, \quad \text { if } Q(t, z)>e^{-\kappa(T-t)} \alpha, \\
Q(T, z)=\alpha \quad \text { and } \quad \frac{\partial Q}{\partial z}(t, 1)=Q(t, 1), \quad \forall t \in[0, T],
\end{array}\right.
$$

on region $\widetilde{\mathcal{R}} \equiv\{(t, z) \mid 0 \leq t \leq T, 0<z \leq 1\}$. 
Define the free boundary $b^{\star}(t)$ of VI 3 as

$$
b^{\star}(t)=\frac{B^{\star}(t, M)}{M} .
$$

Then $b^{\star}(t)$ satisfies the following smooth-pasting conditions:

$$
\begin{aligned}
& Q\left(t, b^{\star}(t)\right)=e^{-\kappa(T-t)} \alpha, \\
& \frac{\partial Q}{\partial z}\left(t, b^{\star}(t)\right)=0 .
\end{aligned}
$$

In terms of the value function $Q(t, z)$, the continuation region $\mathbf{C}^{\star \star}$ and the surrender region $\mathbf{S}^{\star \star}$ of VI 3 are given by

$$
\begin{aligned}
\mathbf{C}^{\star \star} & =\left\{(t, z) \in \widetilde{\mathcal{R}} \mid Q(t, z)>e^{-\kappa(T-t)} \alpha\right\} \\
& =\left\{(t, z) \in \widetilde{\mathcal{R}} \mid b^{\star}(t)<z<1\right\},
\end{aligned}
$$

and

$$
\begin{aligned}
\mathbf{S}^{\star \star} & =\left\{(t, z) \in \widetilde{\mathcal{R}} \mid Q(t, z)=e^{-\kappa(T-t)} \alpha\right\} \\
& =\left\{(t, z) \in \widetilde{\mathcal{R}} \mid 0<z \leq b^{\star}(t)\right\} .
\end{aligned}
$$

Thus, the the value function $Q(t, z)$ can be seen as the solution of the following nonhomogeneous PDE with the mixed boundary condition:

$$
\begin{aligned}
& \mathcal{L} Q(t, z)=-\alpha(r-\kappa) e^{-\kappa(T-t)} \mathbf{1}_{\left\{x \leq b^{\star}(t)\right\}}, \\
& Q(T, z)=\alpha \quad \text { and } \quad \frac{\partial Q}{\partial z}(t, 1)=Q(t, 1),
\end{aligned}
$$

with smooth-pasting conditions (3), on domain $\widetilde{\mathcal{R}}$.

Remark 3.2 The right-hand side of the equation in the first line of (4) is obtained by substituting $Q(t, z)=e^{-\kappa(T-t)} \alpha$ into $\mathcal{L} Q(t, z)$.

According to Jeon et al. [18], the non-homogeneous PDE (4) with mixed boundary condition for operator $\mathcal{L}$ can be extended to region $\widetilde{\mathcal{R}}^{\text {ext }} \equiv\{(t, z) \mid 0 \leq t \leq T, 0<z<\infty\}$ as follows:

Lemma 3.1 The solution of $Q(t, z)$ of $P D E(4)$ with mixed boundary condition satisfies the following extended PDE in $\widetilde{\mathcal{R}}^{\text {ext: }}$

$$
\begin{aligned}
\mathcal{L} Q(t, z)= & -h(t, z) \mathbf{1}_{\{z<1\}}-h\left(t, \frac{1}{z}\right)\left(\frac{1}{z}\right)^{\left(k_{2}-1\right)} \mathbf{1}_{\{z>1\}} \\
& -\left(k_{2}+1\right) z\left[\int_{1}^{x} h\left(t, \frac{1}{y}\right)\left(\frac{1}{y}\right)^{\left(k_{2}+1\right)} d y\right] \mathbf{1}_{\{z>1\}}, \\
Q(T, z)=\alpha & \left\{\mathbf{1}_{\{z<1\}}+\left(\frac{1}{z}\right)^{\left(k_{2}-1\right)} \mathbf{1}_{\{z>1\}}+\left(k_{2}+1\right) z\left[\int_{1}^{z}\left(\frac{1}{y}\right)^{\left(k_{2}+1\right)} d y\right] \mathbf{1}_{\{x>1\}}\right\},
\end{aligned}
$$

where $h(t, z)=\alpha(r-\kappa) e^{-\kappa(T-t)} \mathbf{1}_{\left\{z>b^{\star}(t)\right\}}$, and $k_{2}=\frac{2(r-c)}{\sigma^{2}}$. 
Proposition 3.3 The value function $Q(t, z)$ defined in VI 3 is represented by

$$
Q(t, z)=Q_{E}(t, z)+Q_{P}(t, z)
$$

where

$$
\begin{aligned}
Q_{E}(t, z)= & \alpha e^{-r(T-t)} \mathcal{N}\left(-d_{-}(T-t, z)\right)-\alpha \frac{1}{k_{2}} e^{-r(T-t)}\left(\frac{1}{z}\right)^{\left(k_{2}-1\right)} \mathcal{N}\left(-d_{-}\left(T-t, \frac{1}{z}\right)\right) \\
& +\alpha\left(1+\frac{1}{k_{2}}\right) z e^{-c(T-t)} \mathcal{N}\left(d_{+}(T-t, z)\right)
\end{aligned}
$$

and

$$
\begin{aligned}
& Q_{P}(t, z) \\
& \qquad \begin{array}{c}
\alpha(r-\kappa) e^{-\kappa(T-t)} \int_{t}^{T} e^{-(r-\kappa)(\eta-t)} \mathcal{N}\left(-d_{-}\left(\eta-t, \frac{x}{x^{*}(\eta)}\right)\right) d \eta \\
\quad-\alpha(r-\kappa) e^{-\kappa(T-t)} \frac{1}{k_{2}}\left(\frac{1}{z}\right)^{\left(k_{2}-1\right)} \int_{t}^{T} e^{-(r-\kappa)(\eta-t)} \mathcal{N}\left(-d_{-}\left(\eta-t, \frac{1}{b^{\star}(\eta) z}\right)\right) d \eta \\
\quad+\alpha(r-\kappa) e^{-\kappa(T-t)}\left(1+\frac{1}{k_{2}}\right) z \int_{t}^{T} b^{\star}(\eta)^{k_{2}} e^{-(c-\kappa)(\eta-t)} \mathcal{N}\left(d_{+}\left(\eta-t, b^{\star}(\eta) z\right)\right) d \eta,
\end{array}
\end{aligned}
$$

where

$$
d_{ \pm}(t, z)=\frac{\log z+\left(r-c \pm \frac{\sigma^{2}}{2}\right) t}{\sigma \sqrt{t}} .
$$

Moreover, the free boundary $b^{\star}(t)$ satisfies

$$
Q_{E}\left(t, b^{\star}(t)\right)+Q_{P}\left(t, b^{\star}(t)\right)=\alpha e^{-\kappa(T-t)} .
$$

Proof By Lemma 3.1, $Q(t, z)$ is the solution of

$$
\begin{aligned}
& \mathcal{L} Q(t, z)=-\delta(t, z), \\
& Q(T, z)=\epsilon(z),
\end{aligned}
$$

on domain $\widetilde{\mathcal{R}}$ with

$$
\begin{aligned}
& \delta(t, z)=\alpha(r-\kappa) e^{-\kappa(T-t)}\left(\mathbf{1}_{\left\{z<b^{\star}(t)\right\}}-\frac{1}{k_{2}} z^{1-k_{2}} \mathbf{1}_{\left\{z>\frac{1}{b^{\star}(t)}\right\}}+\left(1+\frac{1}{k_{2}}\right) b^{\star}(t) z \mathbf{1}_{\left\{z>\frac{1}{b^{\star}(t)}\right\}}\right), \\
& \epsilon(z)=\alpha\left(\mathbf{1}_{\{z<1\}}-\frac{1}{k_{2}} z^{1-k_{2}} \mathbf{1}_{\{z>1\}}+\left(1+\frac{1}{k_{2}}\right) z \mathbf{1}_{\{z>1\}}\right) .
\end{aligned}
$$

Then, we can define $\hat{Q}(t, w)$ as the Mellin transform of $Q(t, z)$.

$$
\hat{Q}(t, w)=\int_{0}^{\infty} Q(t, z)^{w-1} d w .
$$


In PDE problem (8), $\hat{Q}(t, w)$ becomes the solution of following ordinary differential equation (ODE):

$$
\frac{d \hat{Q}}{d t}+\frac{\sigma^{2}}{2}\left(w^{2}+\left(1-k_{2}\right) w-k_{1}\right) \hat{Q}=-\hat{\delta}(t, w)
$$

where $\hat{\delta}(t, w)$ is the Mellin transform of $\delta(t, z)$, and $k_{1}=\frac{2 r}{\sigma^{2}}, k_{2}=\frac{2(r-c)}{\sigma^{2}}$, and $\mathcal{A}(w)=w^{2}+(1-$ $\left.k_{2}\right) w-k_{1}$. Then, the solution $\hat{Q}(t, w)$ of $\mathrm{ODE}(9)$ is given by

$$
\hat{Q}(t, w)=e^{\frac{1}{2} \sigma^{2} \mathcal{A}(w)(T-t)} \hat{\epsilon}(w)+\int_{t}^{T} e^{\frac{1}{2} \sigma^{2} \mathcal{A}(w)(\eta-t)} \hat{\delta}(\eta, w) d \eta
$$

where $\hat{\epsilon}(w)$ is the Mellin transform of $\epsilon(z)$.

Applying the inverse Mellin transform to (10),

$$
\begin{aligned}
Q(t, z)= & \frac{1}{2 \pi i} \int_{c-i \infty}^{c+i \infty} e^{\frac{1}{2} \sigma^{2} \mathcal{A}(w)(T-t)} \hat{\epsilon}(w) z^{-w} d w \\
& +\frac{1}{2 \pi i} \int_{c-i \infty}^{c+i \infty} \int_{t}^{T} e^{\frac{1}{2} \sigma^{2} \mathcal{A}(w)(\eta-t)} \hat{\delta}(\eta, w) z^{-w} d \eta d w .
\end{aligned}
$$

To calculate (11), we consider

$$
\mathcal{B}(t, z)=\frac{1}{2 \pi i} \int_{c-i \infty}^{c+i \infty} e^{\frac{1}{2} \sigma^{2} \mathcal{A}(w) t} z^{-w} d w
$$

By Sect. 3 in Jeon et al. [18],

$$
\mathcal{B}(t, z)=e^{-\frac{\sigma^{2}}{2}\left\{\left(\frac{1-k_{2}}{2}\right)^{2}+k_{1}\right\} t} \frac{z^{\frac{1-k_{2}}{2}}}{\sigma \sqrt{2 \pi t}} \exp \left\{-\frac{1}{2} \frac{(\log z)^{2}}{\sigma^{2} t}\right\} .
$$

Thus, by the Mellin convolution property stated in Appendix of Jeon et al. [18],

$$
Q(t, z)=\int_{0}^{\infty} \epsilon(u) \mathbf{1}_{\{u<1\}} \mathcal{B}\left(T-t, \frac{z}{u}\right) \frac{1}{u} d u+\int_{t}^{T} \int_{0}^{\infty} \delta(\eta, u) \mathbf{1}_{\{u<1\}} \mathcal{B}\left(\eta-t, \frac{z}{u}\right) \frac{1}{u} d u d \eta
$$

Let

$$
\begin{aligned}
& Q_{E}(t, z)=\int_{0}^{\infty} \epsilon(u) \mathbf{1}_{\{u<1\}} \mathcal{B}\left(T-t, \frac{z}{u}\right) \frac{1}{u} d u, \\
& Q_{P}(t, z)=\int_{t}^{T} \int_{0}^{\infty} \delta(\eta, u) \mathbf{1}_{\{u<1\}} \mathcal{B}\left(\eta-t, \frac{z}{u}\right) \frac{1}{u} d u d \eta .
\end{aligned}
$$

For any $\rho \in \mathbb{R}$ and $\beta>0$, by direct computation, we easily derive the following relations:

$$
\begin{aligned}
& \int_{0}^{\beta} u^{-\rho} \mathcal{B}\left(t, \frac{z}{u}\right) \frac{1}{u} d u=z^{-\rho} e^{-\frac{1}{2} \sigma^{2} t\left\langle\left(\frac{1-k_{2}}{2}\right)^{2}+k_{1}-\left(\frac{1-k_{2}}{2}+\rho\right)^{2}\right\}} \mathcal{N}\left(\frac{-\log \frac{z}{\beta}+\sigma^{2} t\left(\frac{1-k_{2}}{2}+\rho\right)}{\sigma \sqrt{t}}\right) \\
& \int_{\beta}^{\infty} u^{-\rho} \mathcal{B}\left(t, \frac{z}{u}\right) \frac{1}{u} d u=z^{-\rho} e^{-\frac{1}{2} \sigma^{2} t\left\{\left(\frac{1-k_{2}}{2}\right)^{2}+k_{1}-\left(\frac{1-k_{2}}{2}+\rho\right)^{2}\right\}} \mathcal{N}\left(\frac{\log \frac{z}{\beta}-\sigma^{2} t\left(\frac{1-k_{2}}{2}+\rho\right)}{\sigma \sqrt{t}}\right)
\end{aligned}
$$


Hence,

$$
\begin{aligned}
Q_{E}(t, z)= & \alpha \int_{0}^{\infty}\left(\mathbf{1}_{\{u<1\}}-\frac{1}{k_{2}} u^{1-k_{2}} \mathbf{1}_{\{u>1\}}+\left(1+\frac{1}{k_{2}}\right) u \mathbf{1}_{\{u>1\}}\right) \mathcal{B}\left(T-t, \frac{z}{u}\right) \frac{d u}{u} \\
= & \alpha e^{-r(T-t)} \mathcal{N}\left(-d_{-}(T-t, z)\right)-\alpha \frac{1}{k_{2}} e^{-r(T-t)}\left(\frac{1}{z}\right)^{\left(k_{2}-1\right)} \mathcal{N}\left(-d_{-}\left(T-t, \frac{1}{z}\right)\right) \\
& +\alpha\left(1+\frac{1}{k_{2}}\right) z e^{-c(T-t)} \mathcal{N}\left(d_{+}(T-t, z)\right)
\end{aligned}
$$

where

$$
d_{ \pm}(t, z)=\frac{\log z+\left(r-c \pm \frac{\sigma^{2}}{2}\right) t}{\sigma \sqrt{t}} .
$$

Similarly,

$$
\begin{aligned}
Q_{P}(t, z)= & \alpha(r-\kappa) e^{-\kappa(T-t)} \int_{t}^{T} \int_{0}^{\infty} e^{\kappa(\eta-t)}\left(\mathbf{1}_{\left\{u<b^{\star}(\eta)\right\}}+\mathbf{1}_{\left\{u>\frac{1}{b^{\star}(\eta)}\right\}}\left(\frac{1}{u}\right)^{\left(k_{2}-1\right)}\right. \\
& \left.-\left(1+\frac{1}{k_{2}}\right)\left[u^{\left(1-k_{2}\right)}-b^{\star}(\eta)^{k 2} u\right] \mathbf{1}_{\left\{u>\frac{1}{b^{\star}(\eta)}\right\}}\right) \mathcal{B}\left(\eta-t, \frac{z}{u}\right) \frac{1}{u} d u d \eta \\
= & \alpha(r-\kappa) e^{-\kappa(T-t)} \int_{t}^{T} e^{-(r-\kappa)(\eta-t)} \mathcal{N}\left(-d_{-}\left(\eta-t, \frac{x}{x^{*}(\eta)}\right)\right) d \eta \\
& -\alpha(r-\kappa) e^{-\kappa(T-t)} \frac{1}{k_{2}}\left(\frac{1}{z}\right)^{\left(k_{2}-1\right)} \int_{t}^{T} e^{-(r-\kappa)(\eta-t)} \mathcal{N}\left(-d_{-}\left(\eta-t, \frac{1}{b^{\star}(\eta) z}\right)\right) d \eta \\
& +\alpha(r-\kappa) e^{-\kappa(T-t)}\left(1+\frac{1}{k_{2}}\right) z \int_{t}^{T} b^{\star}(\eta)^{k_{2}} e^{-(c-\kappa)(\eta-t)} \mathcal{N}\left(d_{+}\left(\eta-t, b^{\star}(\eta) z\right)\right) d \eta .
\end{aligned}
$$

By smooth-pasting conditions in (3), we have obtained the desired result.

From the substitution (2), the solution $V^{\star}(t, f, M)$ of VI 2 is given by

$$
V^{\star}(t, f, M)=M Q\left(t, \frac{f}{M}\right) \quad \text { and } \quad B^{\star}(t, M)=M b^{\star}(t)
$$

By Proposition 3.1, Proposition 3.2, and Proposition 3.3, we can derive the following theorem.

Theorem 3.1 Let $V\left(t, F_{t}, m_{t}\right)$ denote the price at time $t$ of the variable annuity with constant guarantee $G$ at maturity $T$ and a surrender benefit equal to the accumulated lookback payoff $e^{-\kappa(T-t)} \alpha m_{t}$. Then $V\left(t, F_{t}, m_{t}\right)$ can be decomposed into a corresponding European part $V_{E}\left(t, F_{t}, M_{t}\right)$ and an early surrender premium part $V_{P}\left(t, F_{t}, m_{t}\right)$

$$
V\left(t, F_{t}, m_{t}\right)=V_{E}\left(t, F_{t}, m_{t}\right)+V_{P}\left(t, F_{t}, m_{t}\right)
$$


where

$$
\begin{aligned}
V_{E}( & \left.t, F_{t}, m_{t}\right) \\
= & \max \left(\alpha m_{t}, G\right) e^{-r \tau} \mathcal{N}\left(-d_{-}\left(\tau, \frac{\alpha F_{t}}{\max \left(\alpha m_{t}, G\right)}\right)\right) \\
& -\frac{\max \left(\alpha m_{t}, G\right)}{k_{2}} e^{-r \tau}\left(\frac{\max \left(\alpha m_{t}, G\right)}{\alpha F_{t}}\right)^{\left(k_{2}-1\right)} \mathcal{N}\left(-d_{-}\left(\tau, \frac{\max \left(\alpha m_{t}, G\right)}{\alpha F_{t}}\right)\right) \\
& +\alpha F_{t}\left(1+\frac{1}{k_{2}}\right) e^{-c \tau} \mathcal{N}\left(d_{+}\left(\tau, \frac{\alpha F_{t}}{\max \left(\alpha m_{t}, G\right)}\right)\right), \\
V_{P}( & \left.t, F_{t}, m_{t}\right) \\
= & \max \left(\alpha m_{t}, G\right)(r-\kappa) e^{-\kappa \tau} \int_{0}^{\tau} e^{-(r-\kappa) \xi} \mathcal{N}\left(-d_{-}\left(\xi, \frac{F_{t}}{B\left(t+\tau, m_{t}\right)}\right)\right) d \xi \\
& -\max \left(\alpha m_{t}, G\right)(r-\kappa) e^{-\kappa \tau} \frac{1}{k_{2}}\left(\frac{\max \left(\alpha m_{t}, G\right)}{F_{t}}\right) \\
& \times \int_{0}^{\tau} e^{-(r-\kappa) \xi} \mathcal{N}\left(-d_{-}-1\right) \\
& \left.+\alpha F_{t}(r-\kappa) e^{-\kappa \tau}\left(1+\frac{1}{B\left(t+\tau, m_{t}\right) F_{t}}\right)\right) d \xi \\
& \times \int_{0}^{\tau} B\left(t+\tau, m_{t}\right)^{k_{2}} e^{-(c-\kappa) \xi} \mathcal{N}\left(d_{+}\left(\xi, \frac{B\left(t+\tau, m_{t}\right) F_{t}}{\max \left(\alpha m_{t}, G\right)^{2}}\right)\right) d \xi \\
\text { with } \tau= & T-t \text { and } k_{2}=\frac{2(r-c)}{\sigma^{2}} .
\end{aligned}
$$

Moreover, the optimal surrender boundary $B\left(t, m_{t}\right)$ is given by

$$
B\left(t, m_{t}\right)=B^{\star}\left(t, \max \left(m_{t}, \frac{G}{\alpha}\right)\right)=\max \left(m_{t}, \frac{G}{\alpha}\right) b^{\star}(t),
$$

where $b^{\star}(t)$ is the solution to the integral equation (7).

Theorem 3.2 When time goes to maturity $T$, the optimal surrender boundary $B(t, m)$ converges to $\max \left(m, \frac{G}{\alpha}\right)$, i.e.,

$$
\lim _{t \rightarrow T_{-}} B(t, m)=\max \left(m, \frac{G}{\alpha}\right) .
$$

Proof By Theorem 3.1, it is enough to show that

$$
\lim _{t \rightarrow T-} b^{\star}(t)=1 .
$$

Consider the time-reversed process of $Q(t, z)$ and $b^{\star}(t)$ as follows:

$$
\tilde{Q}(\tau, z)=Q(T-\tau, z) \text { and } \quad \tilde{b}^{\star}(\tau)=b^{\star}(T-t) .
$$

Define the Laplace-Carson transform $Q^{*}$ of $\tilde{Q}$ by

$$
Q^{*}(\lambda, z)=\int_{0}^{\infty} \tilde{Q}(\tau, z) \lambda e^{-\lambda \tau} d \tau .
$$


Also, let

$$
b^{*}(\lambda)=\int_{0}^{\infty} \tilde{b}^{\star}(\tau) e^{-\lambda \tau} d \tau
$$

From VI 3, $\tilde{Q}(\tau, z)$ satisfies the following equation:

$$
\begin{cases}-\frac{\partial \tilde{Q}}{\partial \tau}+\frac{\sigma^{2}}{2} z^{2} \frac{\partial^{2} \tilde{Q}}{\partial z^{2}}+(r-c) z \frac{\partial \tilde{Q}}{\partial z}-r \tilde{Q}=0, & \tilde{b}^{\star}(\tau)<z \leq 1, \\ \tilde{Q}(t, z)=e^{-\kappa \tau} \alpha, \quad \tilde{Q}(T, z)=\alpha, & \tilde{b}^{\star}(\tau) \geq z,\end{cases}
$$

with the mixed boundary condition $\frac{d \tilde{Q}}{d z}(\tau, 1)=\tilde{Q}(\tau, 1)$.

By taking the Laplace-Carson transform to PDE (13), we obtain

$$
\begin{cases}\frac{\sigma^{2}}{2} z^{2} \frac{d^{2} Q^{*}}{d z^{2}}+(r-c) z \frac{d Q^{*}}{d z}-(r+\lambda) Q^{*}+\lambda \alpha=0, & b^{*}(\lambda)<z \leq 1, \\ Q^{*}(\lambda, z)=\frac{\lambda \alpha}{\lambda+\kappa}, & b^{*}(\lambda) \geq z \\ \frac{d Q^{*}}{d z}(\lambda, 1)=Q^{*}(\lambda, 1) . & \end{cases}
$$

By smooth-pasting conditions, at $z=b^{*}(\lambda)$,

$$
Q^{*}\left(\lambda, b^{*}(\lambda)\right)=\frac{\lambda \alpha}{\lambda+\kappa}, \quad \frac{d Q^{*}}{d z}\left(\lambda, b^{*}(\lambda)\right)=0 .
$$

For $z \in\left(b^{*}(\lambda), 1\right]$, we assume a general solution of the form

$$
Q^{*}(\lambda, z)=c_{1}(\lambda)\left(\frac{z}{b^{*}(\lambda)}\right)^{\eta_{1}(\lambda)}+c_{2}(\lambda)\left(\frac{z}{b^{*}(\lambda)}\right)^{\eta_{2}(\lambda)}+\frac{\lambda \alpha}{\lambda+r}
$$

where $\eta_{1}(\lambda)(>1)$ and $\eta_{2}(\lambda)(<0)$ are the roots of the following algebraic equation for given $\lambda$ :

$$
\frac{\sigma^{2}}{2} \eta^{2}+\left(r-c-\frac{\sigma^{2}}{2}\right) \eta-(\lambda+r)=0
$$

By using smooth-pasting conditions (15), we deduce that

$$
c_{1}(\lambda)=\frac{\eta_{2}}{\eta_{2}-\eta_{1}} \frac{\lambda \alpha(r-\kappa)}{(\lambda+r)(\lambda+\kappa)}, \quad c_{2}(\lambda)=-\frac{\eta_{1}}{\eta_{2}-\eta_{1}} \frac{\lambda \alpha(r-\kappa)}{(\lambda+r)(\lambda+\kappa)} .
$$

Using the mixed boundary condition in $(14), b^{*}(\lambda)$ is the solution of the following algebraic equation:

$$
0=\frac{\eta_{1}(\lambda)\left(1-\eta_{2}(\lambda)\right)}{\eta_{2}(\lambda)\left(1-\eta_{1}(\lambda)\right)}\left(b^{*}(\lambda)\right)^{\eta_{1}(\lambda)-\eta_{2}(\lambda)}+\frac{\lambda+\kappa}{r-\kappa} \frac{\eta_{1}(\lambda)-\eta_{2}(\lambda)}{\eta_{2}(\lambda)\left(1-\eta_{1}(\lambda)\right)}\left(b^{*}(\lambda)\right)^{\eta_{1}(\lambda)}-1 .
$$

Let us define $\psi(x ; \lambda)$ as

$$
\psi(x ; \lambda)=\frac{\eta_{1}(\lambda)\left(1-\eta_{2}(\lambda)\right)}{\eta_{2}(\lambda)\left(1-\eta_{1}(\lambda)\right)} x^{\eta_{1}(\lambda)-\eta_{2}(\lambda)}+\frac{\lambda+\kappa}{r-\kappa} \frac{\eta_{1}(\lambda)-\eta_{2}(\lambda)}{\eta_{2}(\lambda)\left(1-\eta_{1}(\lambda)\right)} x^{\eta_{1}(\lambda)}-1 .
$$


We deduce that

$$
\left\{\begin{array}{l}
\psi(0 ; \lambda)=-1<0 \\
\psi(1 ; \lambda)=\frac{\left(\eta_{1}(\lambda)-\eta_{2}(\lambda)\right)}{\eta_{2}(\lambda)\left(1-\eta_{1}(\lambda)\right)}\left(1+\frac{\lambda+\kappa}{r-\kappa}\right)>0 \\
\psi^{\prime}(x ; \lambda)=\frac{\eta_{1}(\lambda)\left((\lambda)(\lambda)-n_{2}(\lambda)\right)}{\eta_{2}(\lambda)\left(1-\eta_{1}(\lambda)\right)} x^{\eta_{1}(\lambda)-1}\left(\left(1-\eta_{2}(\lambda)\right) x^{-\eta_{2}(\lambda)}+\frac{\lambda+\kappa}{r-\kappa}\right)>0 .
\end{array}\right.
$$

Hence, there exists a unique $b^{*}(\lambda) \in(0,1)$ for any $\lambda>0$ such that $\psi\left(b^{*}(\lambda) ; \lambda\right)=0$. This implies that

$$
\lim _{\lambda \rightarrow \infty} b^{*}(\lambda) \leq 1
$$

Let us rewrite the algebraic equation (16) as follows:

$$
\frac{\eta_{1}(\lambda)\left(1-\eta_{2}(\lambda)\right)}{\eta_{2}(\lambda)\left(1-\eta_{1}(\lambda)\right)} e^{\left(\eta_{1}(\lambda)-\eta_{2}(\lambda)\right) \log b^{*}(\lambda)}+\frac{\lambda+\kappa}{r-\kappa} \frac{\eta_{1}(\lambda)-\eta_{2}(\lambda)}{\eta_{2}(\lambda)\left(1-\eta_{1}(\lambda)\right)} e^{\eta_{1}(\lambda) \log b^{*}(\lambda)}=1 .
$$

Since $\eta_{1}(\lambda) \sim O(\sqrt{\lambda})$ and $\eta_{2}(\lambda) \sim O(-\sqrt{\lambda})$ (as $\left.\lambda \rightarrow \infty\right)$, we can deduce that the order of the left-hand side in (18) is

$$
\sqrt{\lambda} e^{\sqrt{\lambda} \log b^{*}(\lambda)}
$$

From (17), if

$$
\lim _{\lambda \rightarrow \infty} b^{*}(\lambda)<1,
$$

it is easy to check that the left-hand side in (18) converges to zero. This is contraction to the right-hand side value of 1 .

That is,

$$
b^{*}(\lambda) \rightarrow 1 \quad \text { as } \lambda \rightarrow \infty .
$$

By virtue of the Abelian theorem on the initial value of the Laplace Transforms, we derive

$$
\lim _{\tau \rightarrow 0} \tilde{b}^{\star}(\tau)=\lim _{\lambda \rightarrow \infty} b^{*}(\lambda)=1
$$

Hence, we have just proved the desired result.

\section{Properties of the optimal surrender strategy}

Figure 2 shows the free boundary $b^{\star}(t)$ (Panel (a) in Fig. 2) and the corresponding optimal surrender boundary $B(t, m)$ (Panel (b) in Fig. 2) when $\sigma=0.1, r=0.05, c^{*}=0.0168, \kappa=0$, $T=15, G=100$, and $\alpha=0.8$. Here, $c^{*}$ denotes the fair fee rate, which is defined as the fee rate $c$ that satisfies the following equation

$$
V\left(0, F_{0}, m_{0}\right)=F_{0} .
$$




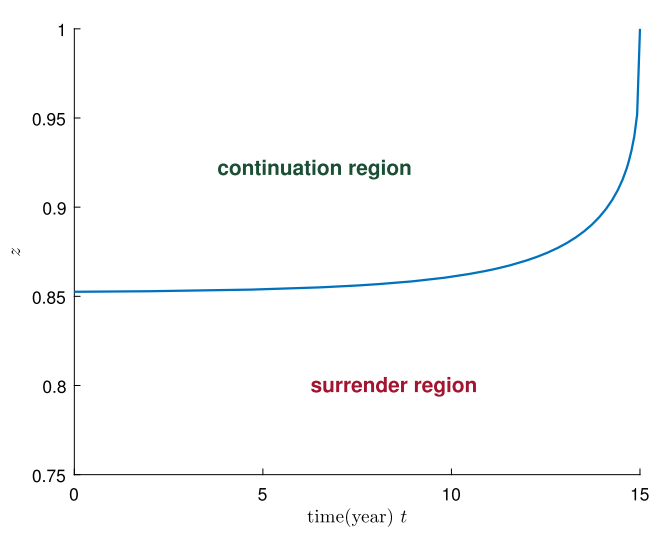

(a) free boundary $b^{\star}(t)$

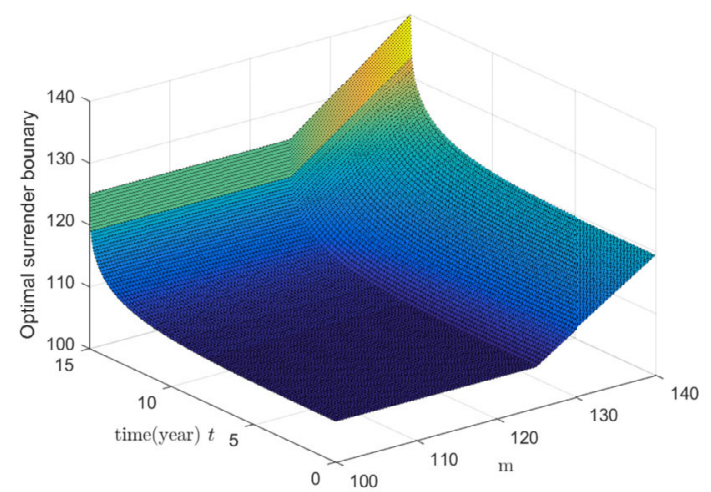

(b) Optimal surreder boundary $B(t, m)$

Figure 2 Parameter values are given by $\sigma=0.1, r=0.05, c^{*}=0.0168, \kappa=0, T=15, G=100$, and $\alpha=0.8$

This means that the fair fee rate $c^{*}$ matches the value of the VA contract at time 0 to the lump-sum premium $F_{0}$ paid at the inception. ${ }^{3}$

Recall from equation (12) that the optimal surrender boundary $B(t, m)$ can be written as

$$
B(t, m)=\max \left(m, \frac{G}{\alpha}\right) b^{\star}(t)
$$

where $b^{\star}(t)$ satisfies the integral equation (7), or equivalently

$$
\frac{Q_{E}\left(t, b^{\star}(t)\right)}{\alpha}+\frac{Q_{P}\left(t, b^{\star}(t)\right)}{\alpha}=e^{-\kappa(T-t)} .
$$

For a given set of parameters, $b^{\star}(t)$ that satisfies (20) can be found by solving the integral equations numerically using the recursive integration method proposed by Huang et al. [14]. From $Q_{E}(t, z)$ and $Q_{P}(t, z)$ in (5) and (6), it can be easily seen that $b^{\star}(t)$, which is determined by equation (20), is irrelevant to $m, G$, and $\alpha$. Thus, regardless of $m, G$, and $\alpha$,

${ }^{3}$ Note that the fair fee rate $c^{*}$ is considered only for Fig. 2 . We set the fee rate $c$ be exogenously given for the other part of this section. 
if we can find $b^{\star}(t)$ from equation (20), then the optimal surrender boundary $B(t, m)$ can be obtained easily as (19). From this, we have the following proposition:

Proposition 4.1 The optimal surrender boundary $B(t, m)$ is increasing in $m, G$, and decreasing in $\alpha$.

Note that our optimal stopping problem (1) can be rewritten as

$$
V(t, f, m)=\alpha \sup _{t \leq \theta \leq T} e^{-r(\theta-t)} \mathbb{E}\left[e^{-\kappa(T-\theta)} \max \left(m_{\theta}, \frac{G}{\alpha}\right) \mid F_{t}=f, m_{t}=m\right] .
$$

Thus, a larger $m$ or $\frac{G}{\alpha}$ results in larger surrender benefit, which induces earlier surrender, or equivalently, higher surrender boundary, and this is exactly what Proposition 4.1 tells us.

We can observe in Panel (a) of Fig. 2 that the free boundary $b^{\star}(t)$ is increasing in time $t$. Note that the continuation region is above the free boundary $b^{\star}(t)$, and the surrender region is below the free boundary $b^{\star}(t)$. Since it is natural that the continuation region shrinks as time goes, $b^{\star}(t)$ must be increasing in time as a consequence. The surface plot for $B(t, m)$ in Panel (b) of Fig. 2 is obtained from $b^{\star}(t)$ in Panel (a) using relation (19). It is straightforward that the optimal surrender boundary is increasing in time. Moreover, as mentioned in Proposition 4.1, $B(t, m)$ is increasing in $m$.

Remark 4.1 In contrast to Bernard et al. [1], in which the surrender region is above the continuation region, there are two surrender boundaries in Jeon and Kwak [5] that introduce the surrender guarantee. However, in our model that considers lookback benefit with surrender guarantee, there is only one surrender boundary, and the surrender region is below the continuation region.

It is obvious from (19) that if some parameter other than $m, G$, and $\alpha$ has a positive impact on $b^{\star}(t)$, then it also has a positive impact on the optimal surrender boundary $B(t, m)$. Thus, we only focus on the effect of parameters $c, \kappa, r$, and $\sigma$ on $b^{\star}(t)$, not $B(t, m)$, throughout the remaining part of this section.

Figure 3 illustrates the effect of fee rate $c$ on the free boundary $b^{\star}(t)$, and we can observe that a higher fee rate results in higher $b^{\star}(t)$, which means earlier surrender. This is an obvious result because the higher fee rate makes the waiting more costly and reduces the time value of the surrender option.

The impact of $\kappa$ on the optimal surrender strategy is shown in Fig. 4, and it shows that the higher $\kappa$, the lower $b^{\star}(t)$, i.e., delayed surrender. Note that early surrender is penalized harder when $\kappa$ is higher. As a result, if $\kappa$ is high, it is better to delay the surrender to avoid a strong penalty.

In Fig. 5, we focus on how the interest rate $r$ affects the optimal surrender strategy. It can be observed that the higher interest rate $r$ results in higher $b^{\star}(t)$ and thus earlier surrender. To understand the intuition behind this result, recall that $b^{\star}(t)$ satisfies the following equation:

$$
Q\left(t, b^{\star}(t)\right)=e^{-\kappa(T-t)} \alpha .
$$




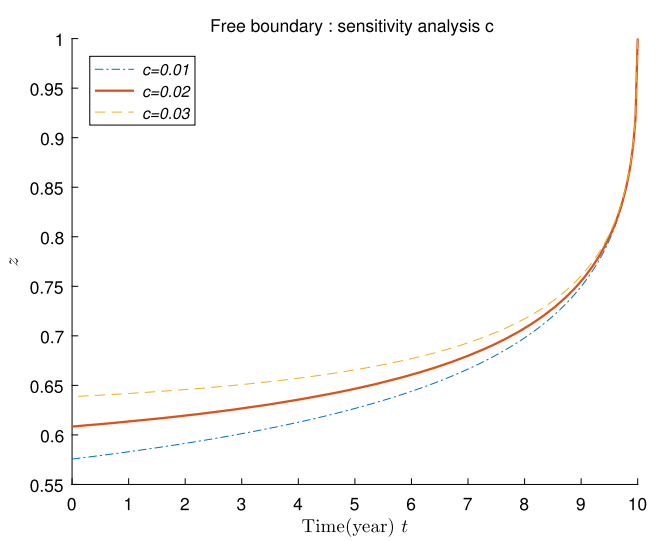

Figure 3 Free boundary $b^{\star}(t)$ : Sensitivity to $c$. Parameter values are given by $\sigma=0.2, r=0.05, \kappa=0, T=10$

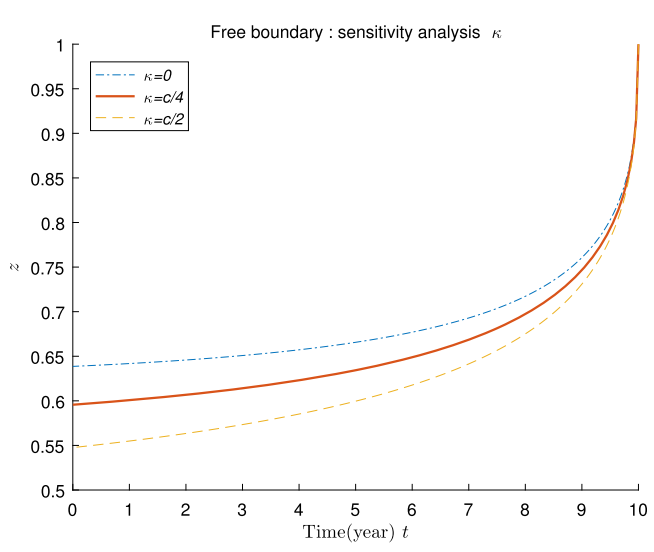

Figure 4 Free boundary $b^{\star}(t)$ : Sensitivity to $\kappa$. Parameter values are given by $\sigma=0.2, r=0.05, c=0.03, T=10$

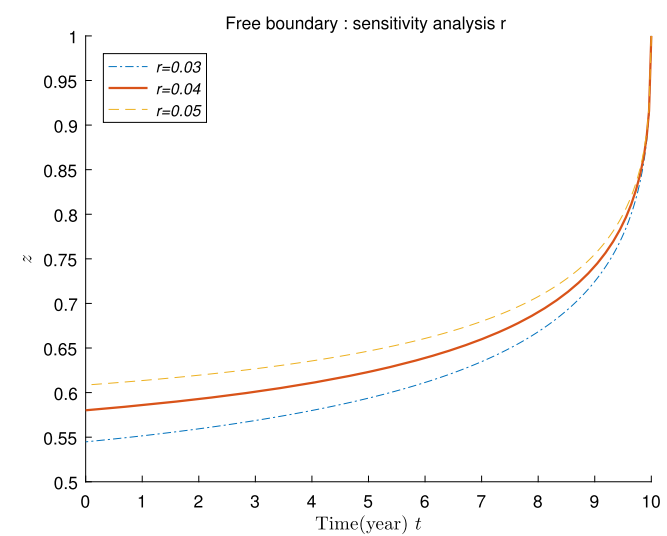

Figure 5 Free boundary $b^{\star}(t)$ : Sensitivity to $r$. Parameter values are given by $\sigma=0.2, c=0.02, \kappa=0, T=10$ 


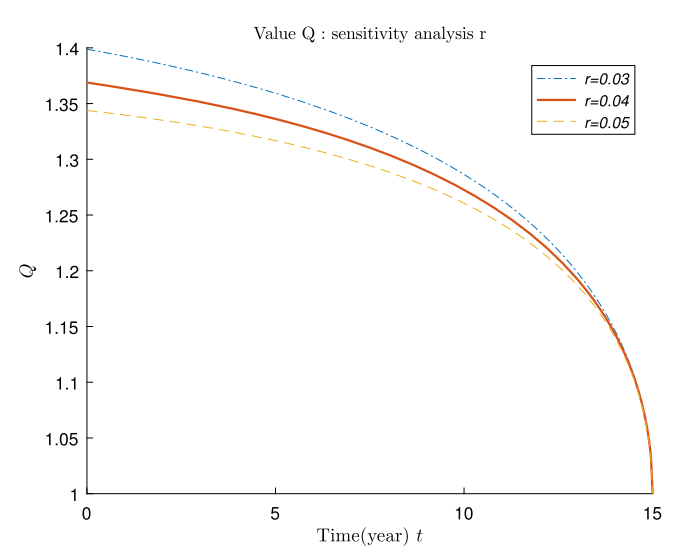

(a) $z=1$

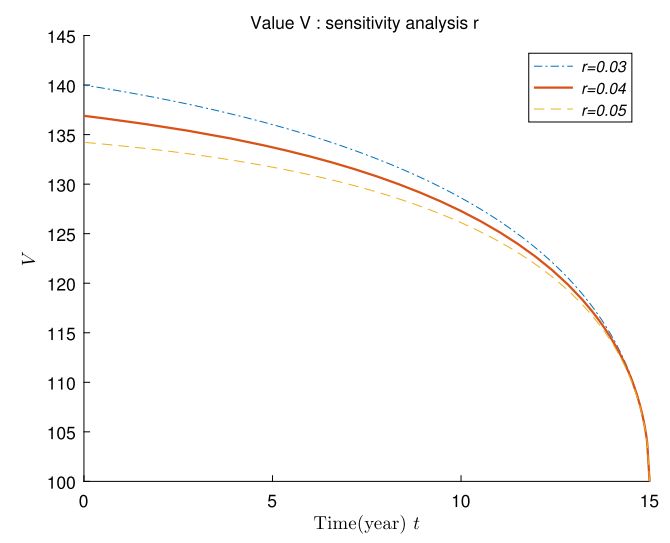

(b) $f=m=G=100$

Figure 6 Value $Q(z)$ and $V(t, f, m)$ : Sensitivity to $r$. Parameter values are given by $\sigma=0.2, c=0.02, \kappa=0$, and $\alpha=1$

In VI 3, $Q(t, \cdot)$ can be interpreted as the value of an option that provides a payoff $e^{-\kappa(T-t)} \alpha$ if it is exercised at time $t$. In this case, the higher interest rate reduces the present value of the option's payoff, and thus, reduces the value of the option $Q(t, \cdot)$. In short, $Q(t, \cdot)$ is decreasing in $r$, and this is shown in Panel (a) of Fig. 6. Since $b^{\star}(t)$ should satisfy the equation (21) and the right-hand side of the equation (21) is not affected by $r$, we can deduce that higher $r$ results in higher $b^{\star}(t)$ as we can see in Fig. 5 .

Remark 4.2 Recall that the relation between $V(t, f, m)$ and $Q(t, z)$ is as follows:

$$
V(t, f, m)=\max \left(m, \frac{G}{\alpha}\right) Q\left(t, \frac{f}{\max \left(m, \frac{G}{\alpha}\right)}\right) .
$$

Thus, the impact of $r$ on $Q(t, z)$ and that on $V(t, f, m)$ are identical as we can see in both panels of Fig. 6. For the same reason, in Fig. 7, we only present the figures for $V(t, f, m)$ instead of showing $Q(t, z)$ and $V(t, f, m)$ together.

Figure 8 shows that higher volatility of the index delays the surrender (lower $\left.b^{\star}(t)\right)$. This result can be explained using an argument similar to that for Fig. 5 . Obviously, the higher 


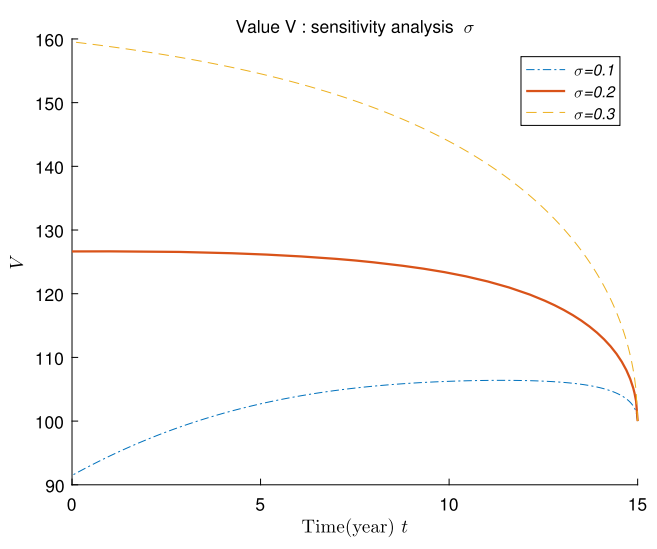

(a) $c=0.03$.

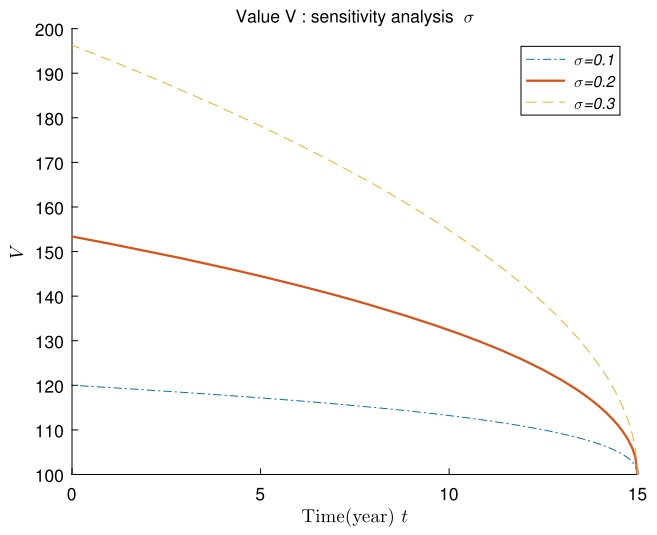

(b) $c=0$.

Figure 7 Value $V(t, f, m)$ : Sensitivity to $\sigma$. Parameter values are given by $r=0.05, \kappa=0, f=100, m=100$ $G=100$, and $\alpha=1$

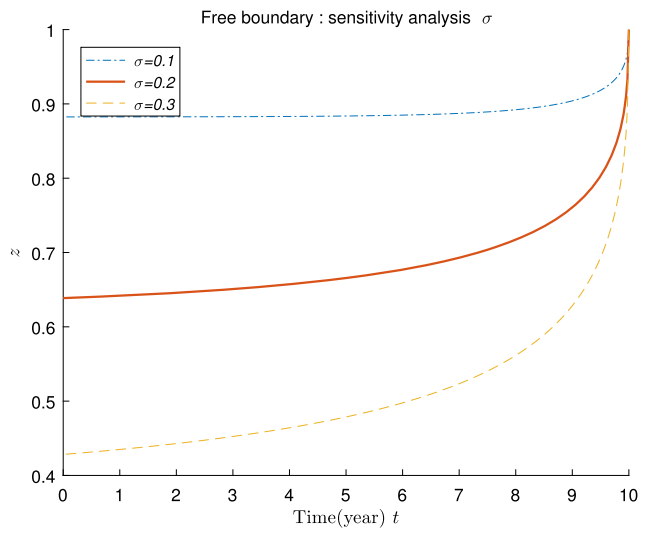

Figure 8 Free boundary $b^{\star}(t)$ : Sensitivity to $\sigma$. Parameter values are given by $r=0.05, c=0.03, \kappa=0, T=10$ 
$\sigma$, the higher $Q(t, \cdot)$, which is the opposite of the impact of $r$. This is consistent with both panels of Fig. 7 . Then, to satisfy the value matching condition in $(21), b^{\star}(t)$ must be decreasing in $\sigma$ as in Fig. 8

Remark 4.3 One may think that something is wrong with Panel (a) in Fig. 7 because the value of the VA contract is increasing in time $t$ during some period of time when $\sigma=0.1$, which never happens for usual financial options. However, this is due to the existence of a positive fee rate $c$. In the VA contract of our model, instead of paying an option premium at the inception, a stream of fee is withdrawn periodically from the policyholder's account as compensation for the guarantees provided by the insurer. In Panel (a) of Fig. 7, $V(t, f, m)$ is calculated for different values of time $t \in[0, T]$, while the fee rate $c$ is fixed as 0.03 . Let $\bar{c}_{t}$ be the fee rate that satisfies $V\left(t, F_{t}, m_{t}\right)=F_{t}$, or equivalently, $V(t, 100,100)=100$ because $F_{t}=100$ and $m_{t}=100$ for all $t$ in Fig. 7. Then, $\bar{c}_{t}$ varies as time goes. If $c$ is positive, then the relation between $\bar{c}_{t}$ and $c$ also changes as time goes, and this may result in the phenomenon in Panel (a) of Fig. 7. However, if $c=0$, then $\bar{c}_{t}$ is always greater than $c$ as in Panel (b) of Fig. 7 , and the value of the VA contract is decreasing in time $t$ like usual financial options.

\section{Concluding remarks}

In this paper, we investigate the optimal surrender policy for VA contract with lookback benefit, which is a proportion of the maximum value of the policyholder's account process. Since we have to find the optimal surrender time for VA contracts, this problem can be categorized into the optimal stopping problem. We obtain its associated two-dimensional parabolic variational inequality that has not been dealt with in the previous research. We convert this problem into the one-dimensional problem and derive the integral equation representation for the optimal surrender boundary. Using recursive integration methods, we present numerical solutions and demonstrate the impacts of the parameters on the optimal surrender strategy. The important characteristic in our model is that the optimal surrender of VA contract with lookback benefit happens when the policyholder's account value decreases to a low enough level and hits the optimal surrender boundary, which is the opposite of the VA contract with a constant guarantee. Therefore, by utilizing our VA contract with lookback benefit, the insurer who has a portfolio of VA contracts will be able to diversify the early surrenders of VA contracts in their portfolio.

Acknowledgements

We are grateful for helpful comments and suggestions from anonymous referees and the editor.

\section{Funding}

Junkee Jeon was supported by Young Research Program through the National Research Foundation of Korea (NRF) funded by the Ministry of Science, ICT \& Future Planning (NRF-2020R1C1C1A01007313). Minsuk Kwak acknowledges that this work was supported by Hankuk University of Foreign Studies Research Fund of 2021. Minsuk Kwak also acknowledges that this work was supported by the National Research Foundation of Korea (NRF) grant funded by the Korea government (MSIT) (NRF-2019R1F1A1062885).

Availability of data and materials

Data sharing not applicable to this article as no datasets were generated or analyzed during the current study.

\section{Declarations}

Competing interests

The authors declare that they have no competing interests. 


\section{Author details}

${ }^{1}$ Department of Applied Mathematics \& Institute of Natural Science, Kyung Hee University, Yongin, Korea. ${ }^{2}$ Department of Mathematics, Hankuk University of Foreign Studies, Yongin, Korea.

\section{Publisher's Note}

Springer Nature remains neutral with regard to jurisdictional claims in published maps and institutional affiliations.

Received: 5 April 2021 Accepted: 22 December 2021 Published online: 04 January 2022

\section{References}

1. Bernard, C., MacKay, A., Muehlbeyer, M.: Optimal surrender policy for variable annuity guarantees. Insur. Math. Econ. 55, 116-128 (2014)

2. Shen, Y., Sherris, M., Ziveyi, J.: Valuation of guaranteed minimum maturity benefits in variable annuities with surrender options. Insur. Math. Econ. 69, 127-137 (2016)

3. Kang, B., Ziveyi, J.: Optimal surrender of guaranteed minimum maturity benefits under stochastic volatility and interest rates. Insur. Math. Econ. 79, 43-56 (2018)

4. Jeon, J., Kwak, M.: Optimal surrender strategies and valuations of path-dependent guarantees in variable annuities. Insur. Math. Econ. 83, 93-109 (2018)

5. Jeon, J., Kwak, M.: Pricing variable annuity with surrender guarantee. J. Comput. Appl. Math. 393, 113508 (2021)

6. Jolaoso, L.O., Shehu, Y., Yao, J.C.: Inertial extragradient type method for mixed variational inequalities without monotonicity. Math. Comput. Simul. 192, 353-369 (2022)

7. Liu, L., Cho, S.Y., Yao, J.C.: Convergence analysis of an inertial Tseng's extragradient algorithm for solving pseudomonotone variational inequalities and applications. J. Nonlinear Var. Anal. 5, 627-644 (2021)

8. Peskir, G.: The Russian option: finite horizon. Finance Stoch. 9, 251-267 (2005)

9. Dai, M., Kwok, Y.K.: American options with lookback payoff. SIAM J. Appl. Math. 66, 206-227 (2005)

10. Bian, B., Hao, C., Xu, H.K., Yuan, Q.: Free boundary and retirement benefits pricing in a jump-diffusion model. J. Nonlinear Var. Anal. 5, 353-370 (2021)

11. Carr, P., Jarrow, R., Myneni, R.: Alternative characterizations of American put options. Math. Finance 2, 87-106 (1992)

12. Detemple, J.: American-Style Derivatives: Valuation and Computation. Champman \& Hall/CRC, Boca Raton (2005)

13. Kim, I.: The analytic valuation of American options. Rev. Financ. Stud. 3, 547-572 (1990)

14. Huang, J., Subrahmanyam, M., Yu, G.: Pricing and hedging American options: a recursive integration method. Rev. Financ. Stud. 9, 277-300 (1996)

15. Buchen, P., Konstandatos, O.: A new method of pricing lookback options. Math. Finance 15(2), 245-259 (2005)

16. Friedman, A.: Parabolic variational inequalities in one space dimension and smoothness of the free boundary. J. Funct. Anal. 18(2), 151-176 (1975)

17. Friedman, A.: Variational Principles and Free-Boundary Problems. Wiley, New York (1982)

18. Jeon, J., Han, H., Kim, H., Kang, M.: An integral equation representation approach for valuing Russian options with a finite time horizon. Commun. Nonlinear Sci. Numer. Simul. 36, 496-516 (2016)

\section{Submit your manuscript to a SpringerOpen ${ }^{\circ}$ journal and benefit from:}

- Convenient online submission

- Rigorous peer review

- Open access: articles freely available online

- High visibility within the field

Retaining the copyright to your article

Submit your next manuscript at $\boldsymbol{\triangleright}$ springeropen.com 